\title{
NEUROSCIENCE
}

\section{The noses have it: murine brain development starts with a smell}

Gretenkord, S. et al. PLoS Biol 17, e2006994 (2019)

When mice are born, they can't see, hear, or discriminate between textures with their whiskers. They can, however, smell. Mouse pups enter the world with a well-developed olfactory bulb (OB), the region of the brain that receives and processes different scents. Without a sense of smell, newborn mice wouldn't be able to find their mother's teat to feed. The brain might be ready, but the behavior is not an innate one-mice still have to learn what their mothers smell like and to associate her particular scent with what they should follow to find a meal.

That association is one of the first things a neonatal mouse learns, says Sabine Gretenkord, a postdoctoral researcher in Ileana Hanganu-Opatz's lab at University Medical Center HamburgEppendorf in Germany. The HanganuOpatz lab studies brain development in rodent models, with a particular interest in the origins of higher level cognition. They usually focus on areas of the limbic system that are associated with memory and decision making, such as the hippocampus and prefrontal cortex, and they want to understand how those areas start to communicate with each other early in life. The olfactory system, functional from birth and the only way through which mice can interact with their environment initially, seemed like a promising place to start looking for clues, says Gretenkord.

In a recent publication in PLoS Biology, they pick up the scent trail into the brain. "The authors have used a really impressive combination of techniques here, including some amazingly challenging electrophysiological recordings in mouse pups in vivo," comments Matthew Grubb, a developmental neurobiologist at King's College London. "It all comes together to characterize the influence on neonatal limbic circuit activity (specifically lateral entorhinal cortex, or LEC) of activity in a rather distant part of the brain-the olfactory bulb."

The techniques used in the paper are well established in adult animals, but the size of their subjects presents unique challenges, explains Gretenkord. Over the past few years, the team has been miniaturizing

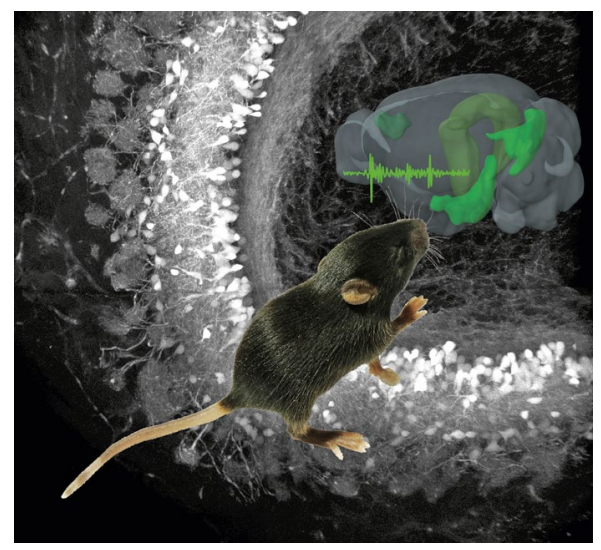

Well developed in the mouse at birth, the olfactory system in pups is linked to regions of the brain involved in behavior and cognition and may be an early driver of brain development. Credit: Hanganu-Opatz Lab and M. Spehr

the equipment needed to monitor brain signals-things like head stages and electrodes have been shrunk and placement adapted for the pups' small size relative to an adult mouse. They've also been working with faster ways to get genetic tools, like those to manipulate neurons via light (known as optogenetics), in to place. In the past, it could take weeks before a mouse would be ready. For this study, they used an approach that took effect within just a few days. "For investigation of the first days of postnatal development, only such fast tools are suitable," says Gretenkord.

With pup-appropriate methods in place, the researchers started this study with some basic questions about anatomical connectivity and electrical activity. "For adult mice, the connectivity patterns are known, but for the age we record, it might be different," Gretenkord says. Some electrical activity in the pups already resembles that of adult mice, with a continuous respiration-related rhythm that depends on nasal air flow. The researchers also observed discontinuous theta-burst activity that is typical for the developing brain. With genetic tagging and tracing, they also confirmed that in the neonatal mice, axons coming from cells in the $\mathrm{OB}$ project to the
LEC, itself the entryway into the limbic system and the cortical areas associated with cognition, and vice versa.

The team then started more deliberate manipulations to see how the two regions interacted with each other. When they inhibited activity in the OB, activity declined in the LEC; when they used optogenetics to generate specific electrical rhythms in the $\mathrm{OB}$, the same rhythms spread to the LEC. "The olfactory bulb is driving the lateral entorhinal cortex," says Gretenkord. And what drives the olfactory bulb? Odors-even in such young animals, presenting them with different scents prompted both slow and fast oscillations in the $\mathrm{OB}$ that spread to the LEC.

"Not only is this functional, in vivo evidence for the influence of one brain region on another during development, it's actually also some of the first characterization of odor-driven activity in newborns," comments Grubb. "The authors suggest that OB-LEC activity might be important in the development of limbic and other circuitry in the brain, and so that's what they need to demonstrate next! But, while olfactory-driven behaviour is crucial for mouse survival right from birth, all of the data here came from animals that are slightly older, at just over 1-week post-birth, and we know there are a lot of changes happening in the $\mathrm{OB}$ and olfactory system in even that first postnatal week. So it would also be interesting to know whether this OB-LEC coupling is also present even earlier, and whether it might then play an even more fundamental role in postnatal brain development."

The scent trail leads on. Gretenkord and her colleagues are planning additional studies on how early odor inputs and interactions with the mother influence broader development over time. "We want to look at what happens if you somehow disrupt this odor input early on, to see if that has an effect on cognitive function in older animals," she says.

\section{Ellen P. Neff}

Published online: 11 March 2019

https://doi.org/10.1038/s41684-019-0272-3 